\title{
A Review on Research Challenges, Limitations and Practical Solutions for Underwater Wireless Power Transfer
}

\author{
Syed Agha Hassnain Mohsan ${ }^{1}$ \\ Ocean College \\ Zhejiang University \\ Zhoushan, China
}

\author{
Asad Islam ${ }^{2}$ \\ School of Energy and Power \\ Engineering, Beihang University \\ Beijing, China
}

\author{
Mushtaq Ali Khan ${ }^{3}$ \\ Department of Marine Science \\ Ocean College, Zhejiang University \\ Zhoushan, China \\ Arfan Mahmood ${ }^{4}$ \\ Complex Networks and Control Lab \\ Shanghai Jiao Tong University \\ Shanghai, China
}

\author{
Laraba Selsabil Rokia ${ }^{5}$ \\ Department of Biomaterials \\ East China University of Science and \\ Technology, Shanghai, China
}

\author{
Alireza Mazinani ${ }^{6}$ \\ School of Electronic and Information \\ Engineering, Beihang University \\ Beijing, China
}

\author{
Hussain Amjad ${ }^{7}$ \\ Department of Marine Science and Information Technology \\ Ocean College, Zhejiang University \\ Zhoushan, China
}

\begin{abstract}
Wireless power transmission is the process to transmit electrical energy without using wire or any physical link. WPT is mainly used at such places where it is difficult to transfer energy using conventional wires. In this research work, we investigated the need and feasibility of wireless power transmission for underwater applications. This research paper will outline research challenges, limitations and practical consideration for underwater wireless power transfer (UWPT). Recent researchers have focused on WPT in air. However, WPT is still a challenging task in underwater environment. In this study, we have presented a review on previous research works in underwater wireless power transfer (UWPT). We have provided a comparison of different techniques implemented for wireless power transfer. This paper also proposes the idea of MIMO wireless power transmission for AUVs charging. This paper elaborates capabilities and limitations of the wireless power transfer system in underwater media as stochastic nature of ocean is a big challenge in wireless power transmission. We have also addressed design challenges and seawater effects on UWPT system.
\end{abstract}

Keywords-Underwater wireless power transfer; charging; MIMO; AUV

\section{INTRODUCTION}

The ongoing research and fast-expanding market of electrical vehicles has reinvigorated the wireless power transfer (WPT) technology. A crucial need of WPT has emerged lately in distributed ocean systems which require excessive power such as ocean monitoring devices, sensor nodes, autonomous underwater vehicle (AUVs). These underwater devices are being used in innumerous underwater applications such as data collection, monitoring and underwater observation. Usually these electronic devices and sensors are deployed in AUVs and ROVs. However, AUVs are advantageous as they do not require any support vessel for ongoing operations. The main disadvantages are its mission time and range which severely limited because of low battery endurance. The typical process to recharge these electronic devices is time-consuming which results into disrupted service and limited operation range. To bridge these gaps, an extensive research work on AUVs is required. Despite of possibility of WPT system for AUVs, still it is bulky in structure which makes it inadequate for smaller AUVs. Nonetheless, overcoming these challenges can unlock a new era of research and new possibilities, offering AUVs to extend operation range and lifetime. A possible solution is to design an underwater docking station. The practical consideration behind this improvement is that the deployed WPT system is also autonomous. In fact, such system has high maintenance periodicity. It also requires high cost for maintenance and useful for near shore applications only. These implementations can be used for specific missions such as SARS operations, oceanography studies, weather reporting and monitoring of water parameters. Here, WPT emerges as a potent solution. Recent scholars are mainly focusing on terrestrial based application of WPT. Several effective techniques have been carried out to maximize WPT system efficiency such as frequency tuning, intermediate field repeaters and impedance matching. In [1], Niu et al. have used metamaterial to enhance WPT system efficiency in air medium. However, WPT technology in underwater environment, where seawater is a challenging medium, is still looking for interest from research fraternity. We have summarized various challenges for underwater wireless power transfer (UWPT) below. 
- What is the effect of high conducting water medium on electrical parameters of WPT system?

- How coil radiation resistance is effected by seawater?

- What are the main losses incurred and how to minimize these losses?

- If any loss is highly dependent of frequency then how to select an operating frequency to achieve an efficient wireless power transmission?

Besides these challenging factors, mobility occurs in WPT system due to dynamic nature of seawater which leads to a varying coupling coefficient. Thus, an optimized design is required to curb this possible variability. Another influential factor to surpass due to high losses in undersea and high electrical permittivity. These challenges pose austere limitations to WPT system. Permittivity and permeability [2-3] parameters for underwater medium are different from air medium. Moreover, the controlling techniques involved in WPT system in air medium require a communication link to keep record of system parameters changes which makes these techniques unviable for underwater wireless power transfer (UWPT). So a controlling mechanism without any communication link is crucially important. It is foreseeable that rapid ongoing research will bring promising revolution in this technology. Furthermore, it is expected that MIMO concept will make it possible to charge multiple devices simultaneously

For an efficient power transmission, WPT should fulfill three conditions: (a) high power, (b) large air gap, (c) high efficiency [4]. The efficiency of WPT system also depends on WPT technique. Transfer efficiencies of different WPT techniques for both near and far region is shown in Fig. 1. 70\% to $90 \%$ transfer efficiency can be achieved through inductive coupling while magnetic resonance coupling offers a moderate efficiency of $40 \%$ to $60 \%$. These transfer efficiencies decay with distance. The efficiency of laser and microwave is less than $30 \%$ and it rapidly decrease as the separation distance between transmitter and receiver coils increases.

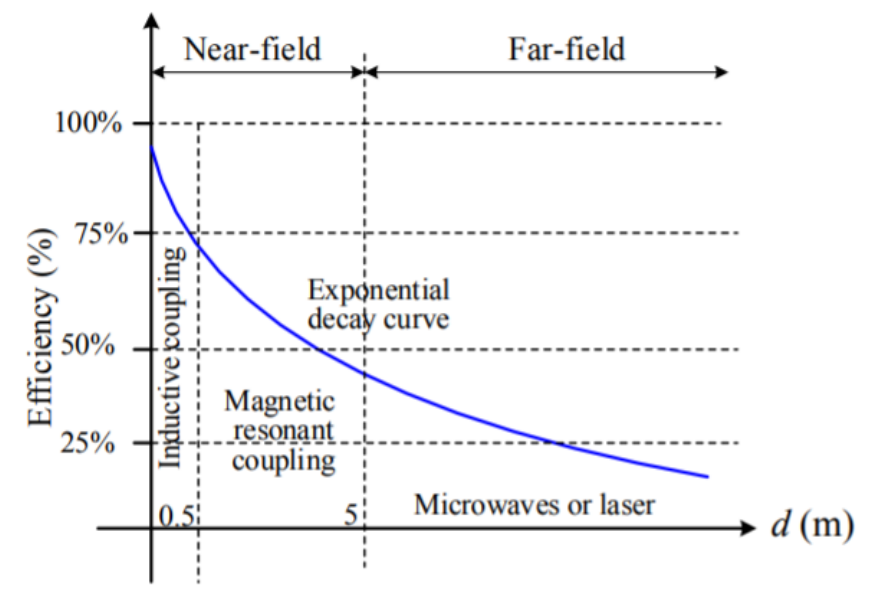

Fig 1. Efficiency for WPT Techniques [5].

\section{RELATED WORK}

Hassnain et al. [6] presented a systematic review on UOWC. They have briefly discussed underwater medium parameters and losses. Numerous researchers have made significant contributions to control load power and enhance efficiency in WPT systems. T. Nguyen et al. [7] have provided a comprehensive survey on EM based WPT technologies for UAVs. C. Cai et al. [8] designed a magnetic coupler for wireless charging of AUVs. Eddy current losses and coil structure to reduce these losses are briefly addressed in [9]. Zheng et al. [10] demonstrated a control mechanism for UWPT system by using artificial neural networks. Zhengchao Yan et al. analyzed eddy current losses under different frequencies and misalignments [11]. IF Lopes et al. [12] have discussed a maximum efficiency tracking strategy for UWPT. A systematic review and critical analysis on UWPT is presented [13]. In [14], Tamura et al. have focused on some pivotal elements to improve coupling coefficients for underwater wireless power transfer (UWPT). In-depth insight is given in [15] to design a WPT system for underwater environment. A comprehensive review of docking methods, AUV energy and AUV IWPT system are discussed in [16]. Researchers have given the best practical overview to design an AUV IWPT system in this article. Table I summarizes an yearly periodical overview of wireless power transfer technology implemented by several research groups.

TABLE I. RESEARCH WORK

\begin{tabular}{|l|l|l|}
\hline Reference \# & Focused Key Challenges & Year \\
\hline Ref. [1] & Frequency Splitting of UWPT & 2016 \\
\hline Ref. [4] & UWPT via Electric Coupling & 2016 \\
\hline Ref. [6] & $\begin{array}{l}\text { EM based WPT Technologies for } \\
\text { UAVs }\end{array}$ & 2020 \\
\hline Ref. [8] & Magnetic Coupler for AUVs Charging & 2020 \\
\hline Ref. [9] & $\begin{array}{l}\text { Coil Structure to Reduce Eddy Current } \\
\text { Losses in WPT }\end{array}$ & 2019 \\
\hline Ref. [10] & $\begin{array}{l}\text { Maximum Efficiency Tracking } \\
\text { Control for UWPT }\end{array}$ & 2020 \\
\hline Ref. [11] & Eddy Current Loss Analysis in UWPT & 2018 \\
\hline Ref. [12] & $\begin{array}{l}\text { Maximum Effciency Tracking } \\
\text { Strategy for UWPT }\end{array}$ & 2020 \\
\hline Ref. [13] & $\begin{array}{l}\text { Systematic review and Critical } \\
\text { Analysis on Wireless Underwater } \\
\text { Power Transfer }\end{array}$ & 2020 \\
\hline Ref. [14] & $\begin{array}{l}\text { Lightweight and High Efficiency } \\
\text { Coupler for UPWT }\end{array}$ & 2019 \\
\hline Ref. [15] & $\begin{array}{l}\text { Maximum Power Efficiency Tracking } \\
\text { for UWPT }\end{array}$ & 2018 \\
\hline Ref. [16] & WPT for AUVs & 2019 \\
\hline Ref. [17] & $\begin{array}{l}\text { Power Transmission Design for } \\
\text { UWPT }\end{array}$ & 2019 \\
\hline Ref. [18] & $\begin{array}{l}\text { Efficient Modeling of UWPT using Z } \\
\text { Parameters }\end{array}$ & 2019 \\
\hline Ref. [19] & $\begin{array}{l}\text { Capacitive WPT for Fresh Water } \\
\text { Operations }\end{array}$ & 2018 \\
\hline Ref. [20] & Load Modulation for UWPT & 2017 \\
\hline Ref. [21] & $\begin{array}{l}\text { UWPT with Curly Coil Structure for } \\
\text { AUVs }\end{array}$ & 2019 \\
\hline Ref. [22] & $\begin{array}{l}\text { Omnidirectional and Positioning } \\
\text { Tolerant AUVs Charging Platform }\end{array}$ & 2019 \\
\hline & [ing \\
\hline
\end{tabular}




\section{WPT METHODS FOR UNDERWATER APPLICATIONS}

The main advantages of wireless charging are reliability, convenience, safety, and a complete automated charging process. These advantages can be obtained through using different techniques. These techniques are classified into two categories: radiative and non-radiative technique. Radiative is RF based while non-radiative is coupling based as we can see in Fig. 2. Fig. 2 also shows that these techniques can be subdivided in four techniques. In radiative technique, power is transferred as an RF wave or optical beam. In non-radiative technique, power is transferred via capacitive charging or EM induction. Furthermore, we have discussed different wireless charging methods for underwater applications.

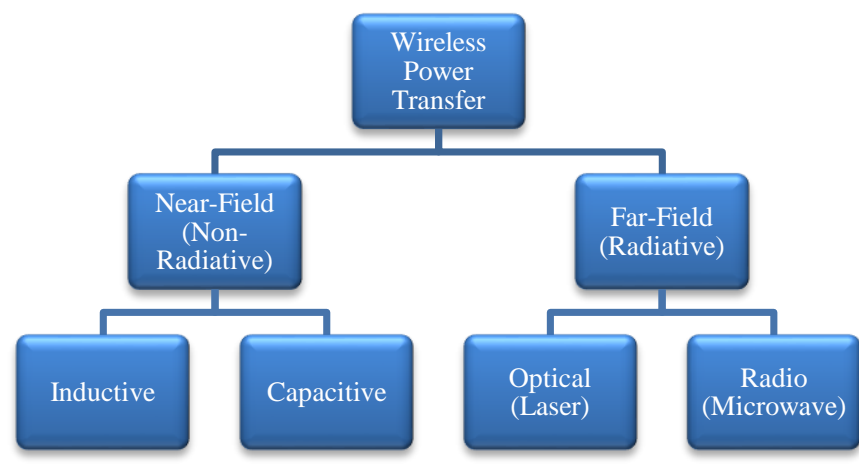

Fig 2. WPT Techniques.

\section{A. Radiative WPT}

Radiative WPT, which is commonly referred as far-field WPT, contains WPT techniques based on lasers or microwaves. Microwaves WPT ranges from $300 \mathrm{MHz}$ to 300 $\mathrm{GHz}$, it allows power transfer at large distance up to few meters. Microwaves WPT systems converts microwave power into DC power through energy-radiating antenna and rectenna. Shinohara et al. in [23] used microwaves WPT in near field by using flat beam pattern. Radio waves face high attenuation at high frequencies in seawater. A few researchers made efforts for underwater wireless power transfer (UWPT) through radio waves but resulted in low efficiency [24]. On the other hand, optical WPT makes use of an LED or LD as transmitter and light photons are detected by PD or solar panel to energy photons into electric signals. Researchers in [25] have successfully implemented blue laser diode for underwater communication and reported data transfer up to $12.4 \mathrm{~Gb} / \mathrm{s}$. However, laser based WPT is not realistic because of its low efficiency and it is also harmful to interface caused by ambient light and biofouling.

\section{B. Nonradiative WPT}

The crucial issue in radiative systems is signal attenuation. The nonradiative WPT systems involve inductive and capacitive power transmission techniques. In this power transmission techniques, the power transfer through magnetic and electric fields is restricted to short distances up to tens of centimeters. The capacitive wireless power transfer contains submerged electrodes which are separated by water medium. In CWPT, water is considered as a lossy dielectric medium. However, CWPT has been neglected due to low coupling capacitance and missing galvanic isolation. A high operation frequency is required in CWPT. In IWPT, the coil diameter or number of turns should be increased to improve efficiency. IWPT system can involve shield materials e.g. ferrite material for better performance. At present, IWPT is more preferable for power transmission in hovering underwater or data transmission on station in seawater.

\section{IWPT}

We will discuss more matured IWPT technology here. IWPT, also considered as inductive wireless power transfer, is WPT based on near-field magnetic coupling through coils.

IWPT is more handy for underwater applications as it is efficient, safe and requires low frequencies as compared to CWPT. In 19th century, Nikola Tesla pioneered IWPT by using EM resonance [26]. He generated resonance for higher voltages to implement WPT to longer distances. The emerging WPT systems have proliferated in recent years by providing matured solutions for charging devices. Nowadays, IWPT systems are commercially available for charging biomedical equipments, electronic gadgets and electric vehicles (EVs). IWPT is efficient power transfer system which is less prone to misalignment between coils. IWPT has been used for underwater robotics [27-29] and ships [30], underwater sensors [31] and AUVs [32-33]. IWPTs eliminate biofouling challenges, DECs, corrosion and potential regarding leakage currents. IWPT saves operational cost for AUVs. Therefore, an extensive research has been carried out from different institutes and leading research groups for development of standards for IWPT. These standards include operating frequency ranges, power levels, design, shielding and many other aspects. A commonly known Qi standard operates at frequencies lower than $250 \mathrm{kHz}$ but its power is limited to $15 \mathrm{~W}$. The AirFuel Resonant standards operate at $6.78 \mathrm{MHz}$ from medical, scientific and industrial band. However, these standards can still be directly implemented to marine applications with high attenuation at higher frequencies in seawater. We have summarized a comparison of different IWPT systems for various applications in Table II and Table III.

TABLE II. COMPARISON OF VARIOUS IWPT SYSTEMS

\begin{tabular}{|c|c|c|c|}
\hline Parameter & $\begin{array}{c}\text { Resonant } \\
\text { IWPT }\end{array}$ & $\begin{array}{c}\text { Loosely } \\
\text { Coupled } \\
\text { Transformer }\end{array}$ & LCWT \\
\hline EMF cancellation & Required & Not required & Not required \\
\hline Air gap & High & Medium & Very low \\
\hline Leakage flux & High & Medium & Low \\
\hline Multiple receiver & Possible & Not possible & Possible \\
\hline Efficiency & Medium & High & Medium \\
\hline $\begin{array}{c}\text { Coupling } \\
\text { coefficient }\end{array}$ & $<0.25$ & $>0.5$ & $\sim 1$ \\
\hline $\begin{array}{c}\text { Misalignment } \\
\text { tolerance }\end{array}$ & High & Medium & Low \\
\hline $\begin{array}{c}\text { Eddy current } \\
\text { losses }\end{array}$ & High & Considerable & Low \\
\hline Hysteresis losses & No & Yes & Yes \\
\hline Copper loss & Low & Low & Very High \\
\hline $\begin{array}{c}\text { Operating } \\
\text { frequency }\end{array}$ & Very high & High & Medium \\
\hline
\end{tabular}


TABLE III. COMPARISON OF VARIOUS IWPT USING RESONANT COILS

\begin{tabular}{|c|c|c|c|c|c|}
\hline Ref. & $\begin{array}{l}\text { Gap } \\
(\mathbf{c m})\end{array}$ & $\begin{array}{c}\text { Efficiency } \\
(\%)\end{array}$ & $\begin{array}{l}\text { Power } \\
\text { Level }\end{array}$ & $\begin{array}{c}\text { Coupling } \\
\text { Coefficient }\end{array}$ & $\begin{array}{c}\text { Experiment } \\
\text { Setup/Applicati } \\
\text { on } \\
\end{array}$ \\
\hline [15] & 4 & 86 & $80 \mathrm{~W}$ & N/A & $\begin{array}{l}\text { Underwater } \\
\text { applications }\end{array}$ \\
\hline [19] & 2 & 80.7 & $400 \mathrm{~W}$ & N/A & AUVs \\
\hline [34] & 7.5 & 80 & N/A & N/A & $\begin{array}{c}\text { Coil submerge in } \\
\text { water tank }\end{array}$ \\
\hline [35] & $\begin{array}{l}20- \\
50\end{array}$ & 97 & $\begin{array}{c}1.1 \\
\text { MW }\end{array}$ & N/A & Charging ferry \\
\hline [36] & 26 & 80 & $3 \mathrm{~kW}$ & N/A & Halogen lamp \\
\hline [37] & 0.9 & 84 & $45 \mathrm{~W}$ & 0.74 & $\begin{array}{c}\text { Underwater } \\
\text { vehicle docking }\end{array}$ \\
\hline [38] & N/A & 85 & $75 \mathrm{~W}$ & N/A & AUV charging \\
\hline [39] & 4 & 82.13 & N/A & 0.25 & $\begin{array}{c}\text { Coils submerge } \\
\text { in water tank }\end{array}$ \\
\hline [40] & N/A & 79 & $200 \mathrm{~W}$ & N/A & AUVs \\
\hline [41] & 0.2 & 70 & $250 \mathrm{~W}$ & N/A & AUVs \\
\hline [42] & 0.5 & 85 & $300 \mathrm{~W}$ & 0.64 & $\begin{array}{c}\text { Ocean } \\
\text { observation }\end{array}$ \\
\hline [43] & 0.2 & 90 & $400 \mathrm{~W}$ & 0.765 & $\begin{array}{c}\text { Deep sea } \\
\text { applications }\end{array}$ \\
\hline [44] & $\begin{array}{c}0.6- \\
1\end{array}$ & 88 & 500 & N/A & AUVs \\
\hline [45] & N/A & 86.19 & 745 & 0.16 & $\begin{array}{l}\text { Leightweight } \\
\text { AUVs }\end{array}$ \\
\hline [46] & 2.1 & 92.41 & $1 \mathrm{~kW}$ & 0.1385 & Charging AUVs \\
\hline [47] & 0.6 & 90 & $1 \mathrm{~kW}$ & 0.64 & $\begin{array}{c}\text { Underwater } \\
\text { vehicles }\end{array}$ \\
\hline [48] & 2.5 & 70.45 & $10 \mathrm{~kW}$ & 0.54 & $\begin{array}{l}\text { Underwater } \\
\text { applications }\end{array}$ \\
\hline [49] & 0.5 & 82 & N/A & 0.43 & AUVs \\
\hline [50] & 8 & 80 & $600 \mathrm{~W}$ & 0.49 & AUVs \\
\hline
\end{tabular}

\section{PRACTICAL CONSIDERATIONS AND RESEARCH CHALLENGES}

Besides all advantages, many technological difficulties exist in WPT system. Like in terms of system modelling, if we increase number of loads then it causes higher order and variable nonlinearities. It is worth considering, different loads have different power requiremetns. Such as desk lamps, mobile phones and computers which involve same WPT surface but their power requirements will be different. Thus, it is very important to guarantee the power requirements of each load. In addition, WPT system characteristics indicate that a remarkable influence on received power exists due to spatial positions of the electronic devices. Finally, new loads can access during operation of multi-load WPT systems. It will decrease power load stability and its efficiency.

Numerous researchers have made significant contributions to control load power and enhance efficiency in multi-load WPT systems. Researchers have involved dynamic controlling impedance matching to realize tunable allocation of power. In [7], scholars have maximized WPT system efficiency through impedance matching in S-S compensation topology of a multiload system.

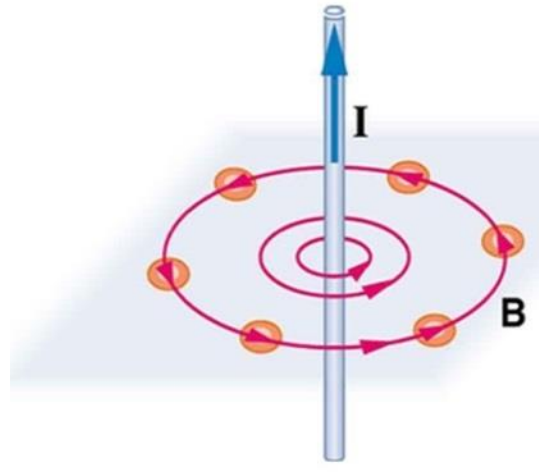

Fig 3. Electromagnetic Induction.

Another challenging factor in WPT systems appears to be distance between transmitter and receiver component. The distance range of WPT plays an essential role in its applications. Wireless power transmission range can be in $m W$ for biomedical implants while for electrical vehicles charging the range is up to $k W$. An intensive research has been carried out to enhance distance range and efficiency of wireless power transfer systems. Our designed experiments follow the theory of electromagnetic induction. When a current $\boldsymbol{i}$ flows through any coil then it creates a magnetic field $\boldsymbol{H}$ around that coil as shown in Fig. 3.

Commonly magnetic field $\mathrm{H}$ is expressed in equation 1 . It is obvious from equations 1 that current intensity is proportional to magnetic field while magnetic field is inversally proportional to the distance from conductor.

$\mathbf{H}=\frac{i}{2 \pi r}$

$\mathbf{B}=\mu \mathbf{H}$

We have plotted magnetic flux density $\boldsymbol{B}$ in MATLAB for different values of current in space by considering equation 2 as shown in Fig. 4. Graph shows a clear decay in magnetic flux density while increasing distance.

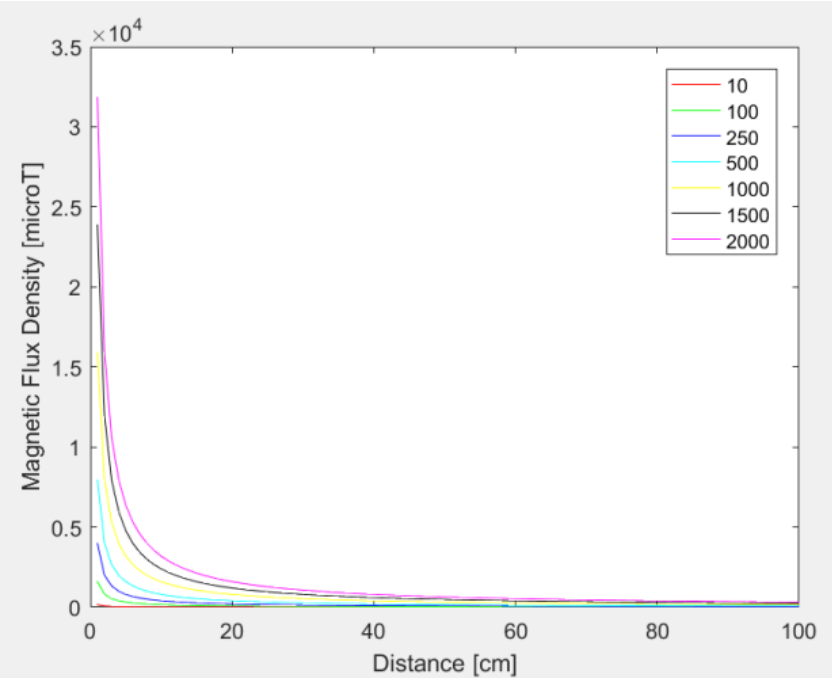

Fig 4. Magnetic Field against Different Currents. 


\section{UNDERWATER COILS CHARACTER}

We have opted circular coils in our system as it requires least material for design and it occupies lease space. Circular coil has less leakage flux and high misalignment tolerance. On the other hand rectangular coil needs more material for design and has more leakage flux. It shows wide variation in coupling when coils are misaligned. Hence, performance of circular coils is better and it is suitable for WPT applications. Characteristic comparison of both types of coils is shown in Table IV.

We have investigated the parasitic capacitance effect by measuring the impedance of coils through impedance analyzer. We checked the shift of its self resonance and coupling coefficient of coils. The coupling coefficient was 0.44 at $20 \mathrm{~mm}$ transmission distance of coils with 15 turns with an inner diameter of $40 \mathrm{~mm}$. We have noticed that coupling coefficient decreases while increasing transmission distance as provided in Fig. 5.

We can conclude from graphs in Fig. 3 that coupling coefficient increases with increasing area and number of turns. Coil winding plays an effective role in efficient coupling [9]. Commonly two ways are adopted to design any coil; flat coil and solenoid coil as shown in Fig. 6.

TABLE IV. COIL CHARACTERISTICS COMPARISON

\begin{tabular}{|l|l|l|}
\hline Parameter & Rectangular Coil & Circular Coil \\
\hline Required Material & More & Less \\
\hline Leakage Flux & More & Less \\
\hline $\begin{array}{l}\text { Leakage Flux } \\
\text { Direction }\end{array}$ & Bottom & Horizontal \\
\hline Horizontal Tolerance & Less & More \\
\hline Vertical Tolerance & More & Less \\
\hline Flux Distribution & Non-uniform & Uniform \\
\hline Polarity & Polarised & Unipolar \\
\hline
\end{tabular}

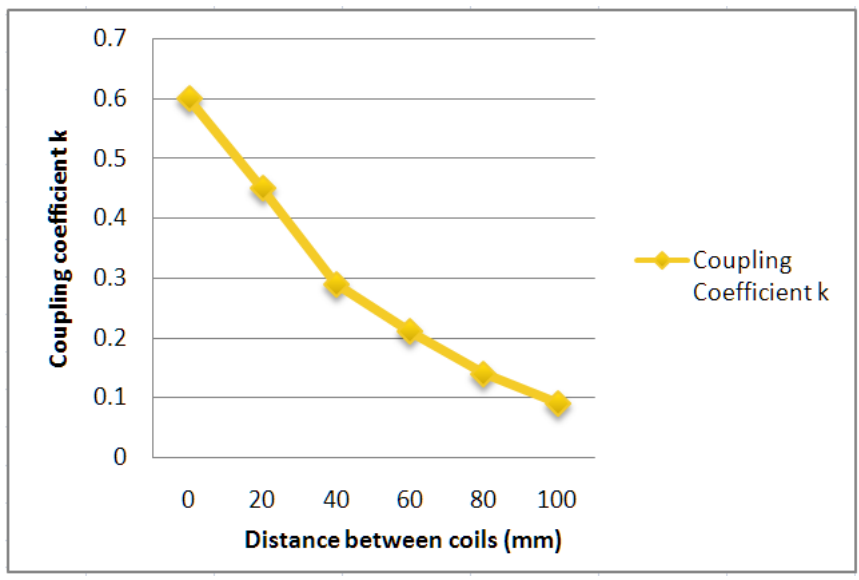

Fig 5. Coupling Coefficient Variation with Distance.

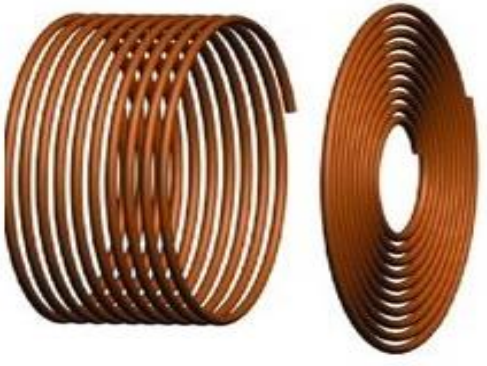

Fig 6. Coil Winding.

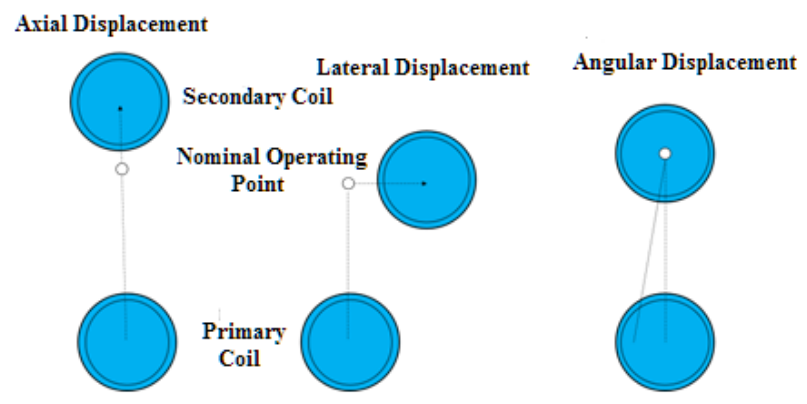

Fig 7. Coil Orientation.

We conclude that mutual inductance has more influence on coupling coefficient than coil selection. The coupling coefficient is strongly connected to the relative distance and orientation between the two coils. Fig. 7 illustrates the three spatial degrees of freedom that affects the coupling coefficient of the system. The exact relationship between the coupling coefficient and the displacement is a complex subject that requires an analysis of the magnetic field strength and shape.

\section{Coil Topology}

It is essential to opt the best suited coil topology for UWPT. In this section we have discussed coil characteristics and design parameters which must be kept into consideration for coil design. We have comparatively investigated the effects of coil shape, size, coupling coefficient, orientation and number of turns. Subsequently, considering both number of turns and change in distance, it is concluded that the coupling coefficient of helix coil is lower than spiral coil. Scholars in [51] have briefly discussed coil topologies. A similar research study in [52] shows that spiral coil gives better efficiency than helix coil as the distance between the coils increases. Surprisingly, the coupling coefficient of spiral coil increases while for helix shape it decreases by increasing number of turns. The coupling coefficient can be referred as an important metric for any coil designing. It shows how much transistor coil flux is captured by the Rx coil. The results presented in Fig. 8 show that the coupling coefficient increases with number of turns. It validates that coil shape has a dramatic effect on coupling. In addition, coil material can also be protected from harsh seawater effects by encapsulating o potting in polyurethane. It has good resistance against seawater effect for a long period of immersion. 


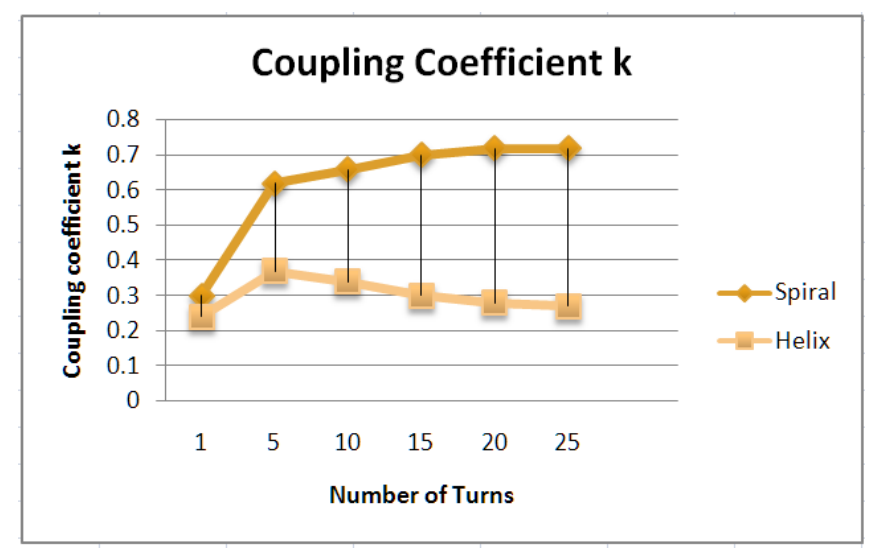

Fig 8. Coupling Coefficient Variation with Coil.

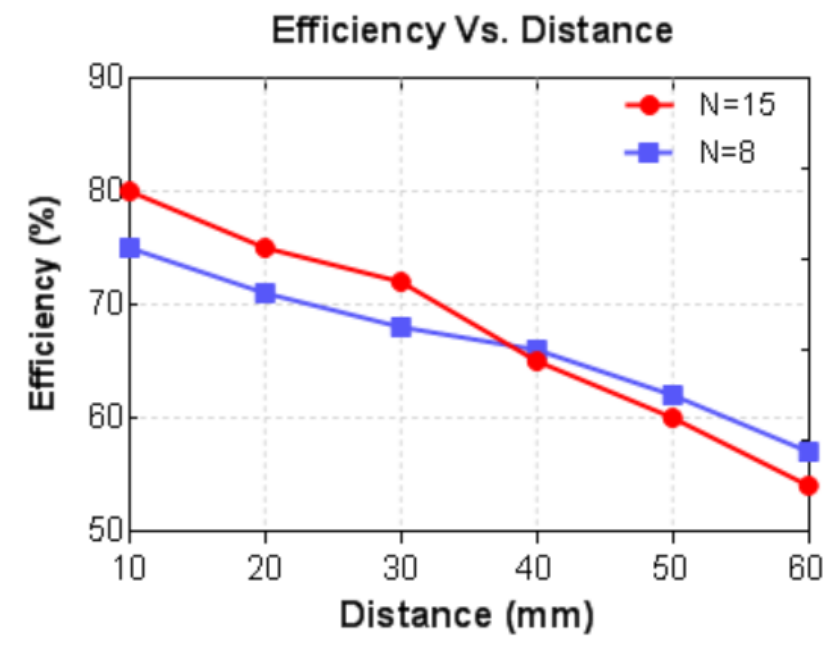

Fig 9. Coil Effect on Efficiency.

In [53], D. Patil et al. have reported that coil with less number of turns gives better performance at longer distances due to decreased reactance. While coil with more number of turns performs better at shorter distances as shown in Fig. 9. Thus, these trade-offs should be kept into consideration for WPT system design.

\section{SEAWATER EFFECTS ON WPT}

We have briefly discussed some effects of seawater on WPT system in this section.

\section{A. Water Conductivity}

The salt in seawater increases conductivity which creates eddy currents [54]. These generated eddy currents oppose magnetic field and reduce field strength of WPT system. It results into decrease in overall efficiency. The loss in proportional to the conductivity. It is concluded that high inductance yields high dependence on conductivity as it generates stronger magnetic field. In addition, it also depends on compensation scheme, if power flows in low voltage, it will generate a stronger magnetic field. It will result into high dependence on water conductivity. Dos Santos et al. in [54] have presented a relation between conductivity and efficiency as shown in Fig. 10.

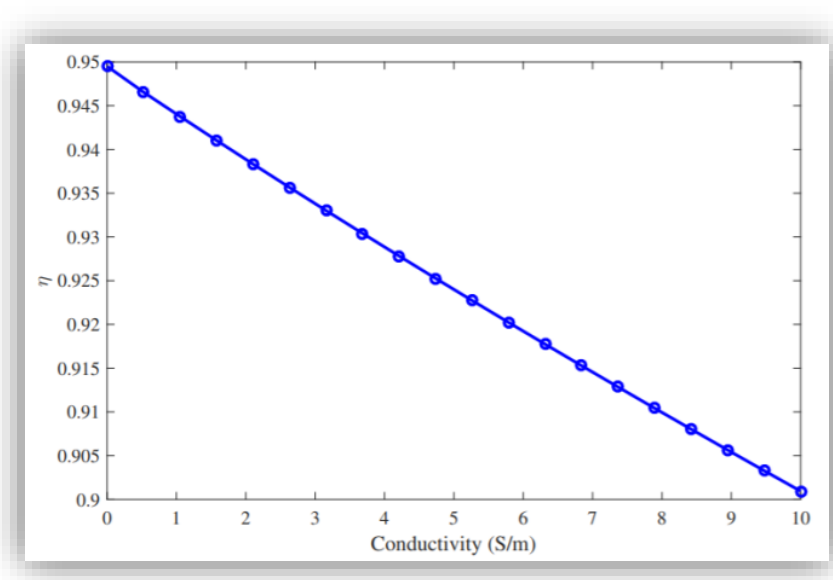

Fig 10. Conductivity Effect on Efficiency [53].

\section{B. OceanCurrents}

The water current can adversely affect WPT system. It can cause misalignments between coils. In an article [47], authors have illustrated that EMF helps to stabilize AUV.

\section{Biofouling}

Another major problem in IWPT systems is biofouling. It s due to amassed marine microorganism on water surface. It can also cause high gap and misalignment between coils, due to which the WPT system efficiency is decreased. Anderson et al. demonstrated in [55] that heat produced in WPT coil can lower the fouling. Antifoul paints and external heating are significantly effective to control pad fouling.

\section{Temperature and Pressure}

Seawater is a cooling medium which can enhance the thermal limit of coils. Researchers in [53] demonstrated that coil power was increased from $600 \mathrm{~W}$ to $1 \mathrm{~kW}$ from air to water medium respectively. The potting material can also effect dissipated heat in coils. High hydrostatic pressure effect on WPT system efficiency is analyzed in [43]. The results show that system efficiency decreases at a depth of $4 \mathrm{~km}$ with $40 \mathrm{MPa}$ pressure. It is due to the decrease in permeability under high pressure which decreases magnetic inductance. Magnetic inductance variation is low as the gap increases. However, authors did not include discussion about eddy current losses in seawater.

\section{VII.ENGINEERING CHALLENGES IN WPT SYSTEMS}

In this section, we have discussed major challenges for IWPT systems in underwater environment.

\section{A. IWPT System for AUV}

The integration of IWPT system on AUV without causing any problem in its shape and hydrodynamic mechanics is essential. The hull, lightweight but mechanically strong, is made of titanium and aluminum. In [37], J. Shi et al. mounted a secondary coil on AUV's hull which generates high magnetic flux. In [50], J.M. Cena et al. placed a secondary coil in AUV's hull which decreased the WPT efficiency. In some earlier works [40-41], [43], authors modified AUV shape which can affect hydrodynamic performance. 


\section{B. Pressure-Tolerant Electronics}

Electronic equipments in AUV's are enclosed in high compressible material in order to tolerate ambient ocean pressure [56]. The material can be solid like urethane or fluid like insulating oil. Pressure tolerant system design is challenging as it involves an intelligent selection of electronic components. Some research groups $[57,58]$ have provided a comprehensive review on selection of pressure tolerant electronics.

\section{Docking Station Stability}

Leveling, orientation and positioning should be maintained while deploying the docking station to seafloor. Research in [59] maintained dock leveling by using heavy railroad wheels. They have introduced additional dumping to overcome inadequate angel effect while employing cone docks. When we employ hooking concept, it can happen that whole docking process should be repeated.

\section{Battery Charging Rate}

The battery operation in dependent on thermal conditions, operation rate and ambient temperature [60]. It is difficult to obtain fast AUV charging behavior as charging and discharging rate is affected by lower ambient temperature. Therefore, commercially available batteries cause delay in mission time. Results discussed in [60] show that increased pressure has a negligible effect on capacity and resistance of battery. While reduced ambient temperature results in decrease in battery capacity and an increase in resistance. Y. Ji et al. [61] have presented a detailed analysis on reduced temperature effect on Li-ion batteries.

\section{CONCLUSION}

The WPT system submerged in seawater has to deal with challenges like corrosive salts, fluctuation and temperature. Thus, a watertight design is required which can halt intrusion and craggy enough to combat waves motion. This section summarizes the critical challenges for underwater wireless power transfer (UWPT). Seawater possesses inherent properties which creates difficulty in EM waves transmission. In addition, there is a rapid increase in radiation resistance on frequencies above $200 \mathrm{kHz}$ which dominates $\mathrm{AC}$ and $\mathrm{DC}$ resistances. It limits operation frequency and it must be kept below threshold in order to achieve good efficiency. Another pivotal constraint is physical dimension of transceiver coils. Larger coils can be cumbersome to integrate while smaller coils can limit the throughput power. Researchers need to find an optimized design through proper simulation and experimental trials.

\section{LIMITATIONS}

One major limitation in UWPT is misalignment between transmitter and receiver coils. Though stagger-tuning technique is implemented in some applications but UWPT still faces limitation due to variation in marine environment. This feature of lateral or angular misalignment between coils can cause in reduction in output voltage. Therefore, optimum frequency for operation is required while achieving a high WPT efficiency. Moreover, research community should investigate optimization and coordination of WPT system in future. MIMO WPT technology can also necessitate power management.

\section{DISCUSSION}

The UWPT system has to face several challenging factors like pressure, temperature and ocean currents. Seawater characteristics also put limit over EM waves transfer. In addition, an essential element is WPT system coil which can affect the transfer efficiency. An appropriate coil topology and accurate orientation is required for better performance. Thus, a proper engineering design is required to handle these challenges. Research community should focus on UWPT system design optimization through proper experimental tests.

\section{FutURE DESIGN IMPROVEMENTS}

Underwater environment is dynamic, uncertain and unstructured in nature. As a result, we encounter limitations when we try to deploy any system in this complex environment. Research community is working to tackle these challenges for significant power and data transfer in underwater environment. An adaptive control system can be used to attain maximum efficiency besides frequent change in coupling coefficient due to stochastic nature of seawater. The efficiency of our WPT system can be enhanced by introducing MIMO concept. Introducing MIMO signal processing and optimization in WPT can give promising results. An additional benefit of MIMO will be the distance range and area of charging station. An AUV can approach nearest charging station and can charge itself at any place of charging station as shown in Fig. 11.

In future, we will work to enhance efficiency of our proposed system by using MNZ metamaterials, multiple coil switching techniques and featuring coil size disparity. Analysis of 3D coil and Quasi-Omnidirectional WPT systems are our future research interests.

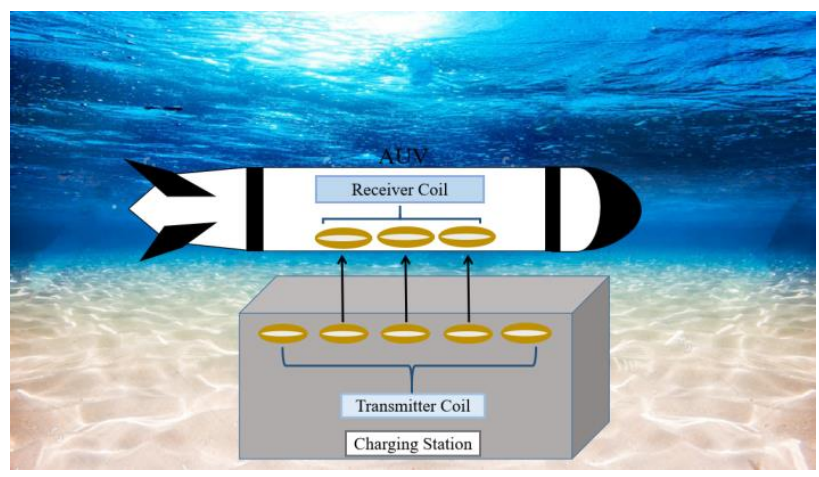

Fig 11. AUVs Self-Charging Station.

\section{REFERENCES}

[1] Niu, Wangqiang, et al. "Frequency splitting of underwater wireless power transfer." 2016 IEEE International Workshop on Electromagnetics: Applications and Student Innovation Competition (iWEM). IEEE, 2016.

[2] Hassnain, S. A., M. J. Mughal, and Q. A. Naqvi. "Layered Chiral Spheres with Zero Backscattering." 2019 Photonics \& Electromagnetics Research Symposium-Fall (PIERS-Fall). IEEE, 2019.

[3] Hassnain, S. A., M. J. Mughal, and Q. A. Naqvi. "Analysis of Effective Medium Parameters on Polarizability of Homogeneous Chiral Sphere." 
2019 Photonics \& Electromagnetics Research Symposium-Fall (PIERSFall). IEEE, 2019.

[4] Urano, Mitsuhiro, and Akira Takahashi. "Study on underwater wireless power transfer via electric coupling." 2016 IEEE International Meeting for Future of Electron Devices, Kansai (IMFEDK). IEEE, 2016.

[5] Jawad, Aqeel Mahmood, et al. "Opportunities and challenges for nearfield wireless power transfer: A review." Energies 10.7 (2017): 1022.

[6] Mehedi, Syed Agha Hassnain Mohsan Md, et al. "A Systematic Review on Practical Considerations, Recent Advances and Research Challenges in Underwater Optical Wireless Communication." IJACSA 11-7 (2020)

[7] T Nguyen, Minh, et al. "Electromagnetic field based wpt technologies for uavs: A comprehensive survey." Electronics 9.3 (2020): 461.

[8] Cai, Chunwei, et al. "A Circumferential Coupled Dipole-Coil Magnetic Coupler for Autonomous Underwater Vehicles Wireless Charging Applications." IEEE Access 8 (2020): 65432-65442.

[9] Zhang, Kehan, et al. "A new coil structure to reduce eddy current loss of WPT systems for underwater vehicles." IEEE Transactions on Vehicular Technology 68.1 (2018): 245-253.

[10] Zheng, Zhongjiu, Ning Wang, and Sara Ahmed. "Maximum efficiency tracking control of underwater wireless power transfer system using artificial neural networks." Proceedings of the Institution of Mechanical Engineers, Part I: Journal of Systems and Control Engineering (2020): 0959651820946510.

[11] Yan, Zhengchao, et al. "Eddy current loss analysis of underwater wireless power transfer systems with misalignments." AIP Advances 8.10 (2018): 101421.

[12] Lopes, Israel Filipe, et al. "Low-Frequency Underwater Wireless Power Transfer: Maximum Efficiency Tracking Strategy." IEEE Latin America Transactions 18.07 (2020): 1200-1208.

[13] Zhou, Jing, et al. "Design considerations for contact-less underwater power delivery: a systematic review and critical analysis." Wireless Power Transfer 7.1 (2020): 76-85.

[14] Tamura, Masaya, Kousuke Murai, and Daiki Fujii. "Lightweight and High-Efficiency Coupler Suitable for Underwater WPT System." 2019 IEEE Asia-Pacific Microwave Conference (APMC). IEEE, 2019.

[15] Orekan, Taofeek, Peng Zhang, and Cyuansi Shih. "Analysis, design, and maximum power-efficiency tracking for undersea wireless power transfer." IEEE Journal of Emerging and Selected Topics in Power Electronics 6.2 (2017): 843-854.

[16] Teeneti, Chakridhar Reddy, et al. "Review of Wireless Charging Systems for Autonomous Underwater Vehicles." IEEE Journal of Oceanic Engineering (2019).

[17] Silva, Miguel, et al. "Power Transmitter Design for Underwater WPT." OCEANS 2019-Marseille. IEEE, 2019.

[18] Kim, Jongwook, et al. "An Efficient Modeling for Underwater Wireless Power Transfer Using Z-Parameters." IEEE Transactions on Electromagnetic Compatibility (2019).

[19] Tamura, Masaya, et al. "Design of a capacitive wireless power transfer system for operation in fresh water." IEEE Transactions on Microwave Theory and Techniques 66.12 (2018): 5873-5884.

[20] Duarte, Candido, et al. "A study on load modulation for underwater wireless power transfer." OCEANS 2017-Aberdeen. IEEE, 2017.

[21] Yan, Zhengchao, et al. "Underwater wireless power transfer system with a curly coil structure for AUVs." IET Power Electronics 12.10 (2019): 2559-2565.

[22] Yang, Canjun, Tianlei Wang, and Yanhu Chen. "Design and analysis of an omnidirectional and positioning tolerant AUV charging platform." IET Power Electronics 12.8 (2019): 2108-2117.

[23] Shinohara, Naoki, and Naoki Kamiyoshikawa. "Study of flat beam in near-field for beam-type wireless power transfer via microwaves." 2017 11th European Conference on Antennas and Propagation (EUCAP). IEEE, 2017.

[24] Naka, Yasumasa, et al. "Improvement in efficiency of underwater wireless power transfer with electric coupling." IEICE Transactions on Electronics 100.10 (2017): 850-857.

[25] Wu, Tsai-Chen, et al. "Blue laser diode enables underwater communication at 12.4 Gbps." Scientific reports 7.1 (2017): 1-10.
[26] Rozman, Matjaz. Inductive wireless power transmission for automotive applications. Diss. Manchester Metropolitan University, 2019.

[27] B. Allotta, L. Pugi, A. Reatti, and F. Corti, "Wireless power recharge for underwater robotics," in Proc. IEEE Int. Conf. Environ. Elect. Eng., IEEE Ind. Commercial Power Syst. Eur., 2017, pp. 1-6.

[28] T. Assaf, C. Stefanini, and P. Dario, "Autonomous underwater biorobots: A wireless system for power transfer," IEEE Robot. Autom. Mag., vol. 20, no. 3, pp. 26-32, Sep. 2013.

[29] H. Jun, A. Asada, T. Ura, Y. Yagita, and Y. Yamauchi, "High speed acoustic network and noncontact power supplier for seafloor geodetic observing robot system," in Proc. OCEANS Conf., 2006, pp. 1-3.

[30] G. Guidi, J. A. Suul, F. Jenset, and I. Sorfonn, "Wireless charging for ships: High-power inductive charging for battery electric and plug-in hybrid vessels," IEEE Electrific. Mag., vol. 5, no. 3, pp. 22-32, Sep. 2017.

[31] D. Yoshioka, H. Sakamoto, Y. Ishihara, T. Matsumoto, and F. Timischl, "Power feeding and data-transmission system using magnetic coupling for an ocean observation mooring buoy," IEEE Trans. Magn., vol. 43, no. 6, pp. 2663-2665, Jun. 2007.

[32] M. D. Feezor, F. Yates Sorrell, and P. R. Blankinship, "An interface system for autonomous undersea vehicles," IEEE J. Ocean. Eng., vol. 26, no. 4, pp. 522-525, Oct. 2001.

[33] T. Orekan and P. Zhang, Underwater Wireless Power Transfer Smart Ocean Energy Converters. New York, NY, USA: Springer, 2019.

[34] A. Askari, R. Stark, J. Curran, D. Rule, and K. Lin, "Underwater wireless power transfer," in Proc. IEEE Wireless Power Transf. Conf., 2015, pp. 1-4.

[35] G. Guidi, J. A. Suul, F. Jenset, and I. Sorfonn, "Wireless charging for ships: High-power inductive charging for battery electric and plug-in hybrid vessels," IEEE Electrific. Mag., vol. 5, no. 3, pp. 22-32, Sep. 2017

[36] M. Kesler and C. Mccarthy, "Highly resonant wireless power transfer in subsea applications," WiTricity, Apr. 2015.

[37] J. Shi, D. Li, and C. Yang, "Design and analysis of an underwater inductive coupling power transfer system for autonomous underwater vehicle docking applications," J. Zhejiang Univ. Sci. C, vol. 15, no. 1, pp. 51-62, Jan. 2014.

[38] V. Bana, M. Kerber, G. Anderson, J. D. Rockway, and A. Phipps, "Underwater wireless power transfer for maritime applications," in Proc. IEEE Wireless Power Transf. Conf., 2015, pp. 1-4.

[39] H. M. Santos, M. R. Pereira, L. M. Pessoa, C. Duarte, and H. M. Salgado, "Assessment of design trade-offs for wireless power transfer on seawater," in Proc. OCEANS Conf., 2016, pp. 1-7.

[40] M. D. Feezor, F. Yates Sorrell, and P. R. Blankinship, "An interface system for autonomous undersea vehicles," IEEE J. Ocean. Eng., vol. 26, no. 4, pp. 522-525, Oct. 2001.

[41] T. McGinnis, C. P. Henze, and K. Conroy, "Inductive power system for autonomous underwater vehicles," in Proc. OCEANS Conf., 2007, pp. $1-5$.

[42] J. Zhou, D. Li, and Y. Chen, "Frequency selection of an inductive contactless power transmission system for ocean observing," Ocean Eng., vol. 60, pp. 175-185, Mar. 2013.

[43] Z. Li, D. Li, L. Lin, and Y. Chen, "Design considerations for electromagnetic couplers in contactless power transmission systems for deep-sea applications," J. Zhejiang Univ. Sci. C, vol. 11, no. 10, pp. 824-834, Oct. 2010.

[44] S.Wang, B. Song, G. Duan, and X. Du, "Automatic wireless power supply system to autonomous underwater vehicles by means of electromagnetic coupler," J. Shanghai Jiaotong Univ., vol. 19, no. 1, pp. 110-114, Feb. 2014.

[45] T. Kan, Y. Zhang, Z. Yan, P. Mercier, and C. C. Mi, "A rotationresilient wireless charging system for lightweight autonomous underwater vehicles," IEEE Trans. Veh. Technol., vol. 67, no. 8, pp. 6935-6942, Aug. 2018.

[46] T. Kan, R. Mai, P. P. Mercier, and C. Mi, "Design and analysis of a threephase wireless charging system for lightweight autonomous underwater vehicles," IEEE Trans. Power Electron., vol. 33, no. 8, pp. 6622-6632, Aug. 2018. 
[47] T. Kojiya, F. Sato, H. Matsuki, and T. Sato, "Construction of noncontacting power feeding system to underwater vehicle utilizing electro magnetic induction," in Proc. Eur. OCEANS Conf., 2005, vol. 1, pp. 709-712.

[48] Z. Cheng, Y. Lei, K. Song, and C. Zhu, "Design and Loss analysis of loosely coupled transformer for an underwater high-power inductive power transfer system," IEEE Trans. Magn., vol. 51, no. 7, pp. 1-10, Jul. 2015.

[49] Z. Yan, K. Zhang, H. Wen, and B. Song, "Research on characteristics of contactless power transmission device for autonomous underwater vehicle," in Proc. OCEANS Conf., 2016, pp. 1-5.

[50] J. M. Cena, "Power transfer efficiency of mutually coupled coils in an aluminum AUV hull," M.S. thesis, , Nav. Postgrad. School, Monterey, CA, USA, 2013.

[51] P. Hadadtehrani, P. Kamalinejad, R. Molavi, and S. Mirabbasi, "On the use of conical helix inductors in wireless power transfer systems," in Proc. IEEE Can. Conf. Elect. Comp. Eng. (CCECE), May 2016, pp. 1-4.

[52] X. Shi et al., "Effects of coil shapes on wireless power transfer via magnetic resonance coupling," J. Electromagn. Waves Appl., vol. 28, no. 11, pp. 1316-1324, 2014.

[53] D. Patil, M. K. McDonough, J. M. Miller, B. Fahimi, and P. T. Balsara, "Wireless power transfer for vehicular applications: Overview and challenges," IEEE Trans. Transp. Electrific., vol. 4, no. 1, pp. 3-37, Mar. 2018.
[54] dos Santos, Hugo Miguel Guedes Pereira. "Underwater Wireless Power Transfer." (2016).

[55] G. Anderson, V. Bana, M. Kerber, A. Phipps, and J. D. Rockway, "Marine fouling and thermal dissipation of undersea wireless power transfer," SPAWAR Syst. Center Pac., San Diego, CA, USA, Tech. Rep. 2056, 2014.

[56] M. Luck et al., "Pressure tolerant systems for deep sea applications," in Proc. OCEANS Conf., 2010, pp. 1-4.

[57] R. P. Granger, C. M. Baer, N. H. Gabriel, J. J. Labosky, and T. C. Galford, "Non-contact wet mateable connectors for power and data transmission," in Proc. OCEANS Conf., 2013, pp. 1-4.

[58] "Seatooth Connect-Wireless Connector," 2015. [Online]. Available: http://www.wfs-tech.com/wpcontent/uploads/2015/06/SeatoothConnect-15-1.0.pdf

[59] B. W. Hobson et al., "The development and ocean testing of an AUV docking station for a 21" AUV," in Proc. OCEANS Conf., 2007, pp. 16.

[60] K. Rutherford and D. Doerffel, "Performance of lithium-polymer cells at high hydrostatic pressure," in Proc. Unmanned Untethered Submersible Technol., 2005.

[61] Y. Ji, Y. Zhang, and C.-Y. Wang, "Li-ion cell operation at low temperatures," J. Electrochem. Soc., vol. 160, no. 4, pp. A636-A649, Feb. 2013. 